\title{
The effect of different rewards under two different conditions on the performance of first-graders ${ }^{\prime, 2}$
}

\author{
Stanley R. Friedman and Norman Thber \\ LOUISИLLE CHILD GUIDANCE CLINIC
}

\begin{abstract}
The acquisition and extinction of a discrimination response as a function of reward and condition was investigated. Each $\mathrm{S}$ was assigned to one of four reward groups, under one of two conditions. The results indicated that magnitude and type of reward do not significantly affect acquisition performance of first-graders, though a differential hierarchy of reward preference is evident in the degree of resistance to extinction.

\section{Problem}

Commenting on the effects of rewards on performance, Hebb $(1955$, p. 250) stated, "...the animal data show that it is not always a matter of extrinsic reward; risk and puzzle can be attractive in themselves, especially for higher animals such as man. If we can accept this, it will no longer be necessary to work out tortuous and improbable ways to explain why human beings work for money, why school children should learn without pain, why a human being in isolation should dislike doing nothing."

Stevenson \& Weir (1959) tested 5-year-old children on a learning task with three schedules of reinforcement; $100 \%$; $66 \%$; and $33 \%$, under conditions of high and low incentive (i.e. magnitude of reward). They found no differences in rate of response under the $100 \%$ condition, but under the other two schedules, the low motivation groups responded significantly more often. It appears that reward becomes an important performance factor only when the uncertainty of the task increases.

After a survey of the reinforcement literature, Lawrence \& Festinger (1962, p. 32) concluded that "The set of conditions that an animal chooses least often in a free choice situation will result in increased resistance to extinction, in a non-choice situation, if the animal has experienced them during acquisition."

Then, if we combine knowledge-of-results (KOR)$100 \%$ schedule of reinforcement-with both low and high levels of reward, we should expect no differences attributable to rewards on acquisition performance, but we would expect both magnitude and type of reward to significantly affect RTE (assuming, of course, that in a free choice situation, Ss would choose high level of reward, and, in addition, would prefer some rewards to others. The second assumption was confirmed in a pilot study). The following experiment was designed to test this dual-hypothesis.

\section{Subjeets}

Eighty first-graders, 39 male and 41 female, from three Louisville public schools served as Ss. The children were predominantly from lower-middle-class families and were selected randomly from the teachers' record-books.

\section{Apparatus}

A three-sided box (open side facing the experimenter) having five doors was used. The front of the box was 18 in high and 36 in wide; the doors were 4 in high and 3 in wide and equally spaced across the width of the box front; they were hinged at the top. The sides of the box were extended back 12 in so as to preclude the possibility of a $\mathrm{S}^{*}$ seeing the rewards being placed behind the doors.

Four rewards were used: small green plastic-tokens; paperclips; small balloons; and gum-drops.

\section{Procedure}

Each $\mathrm{S}$ was assigned randomly to one of the eight combinations of reward and condition (the only restriction being that an equal number of children be assigned to each cell). Two conditions were used, Token and Direct. In the Direct condition, the child was told that each time he opened the correct door, he would find a reward (which was shown to him) and he would be allowed to keep all that he found. In the Token condition, the child was told that each time he opened the correct door he would find a plastic-token, and that if he found enough tokens (no specific amount was stated) he would get to keep on e reward (whichever one of the four reward conditions to which the $S$ was assigned). The reward remained visible to the $S$ throughout the testing session. The "correct" choice response was only door two, the second door from the S's left. Fifty acquisition trials were given, and then the child went through 25 extinction trials, no door being rewarded. The children were switched to extinction without any delay or any other indication that the situation had changed. (The children were not permitted to take the rewards back with them to the classroom. They were told that they would receive it and an additional prize at the end of the school-day if they did not tell anybody about the "game". There was no indication that the children discussed the testing or rewards.)

\section{Instruetions}

"You see this box has five doors, (pointing). Each time you hear the word "GO" you may open any one of the doors you want, but you may only open one door each time you hear the word "GO". If you open the correct door, you'll find a (appropriate reward) behind the door. If you do find a (reward), reach in, take it out, and put it in this box (provided the child). Any questions? Then let's play the game." 


\section{Results}

A model I analysis of variance (Winer, 1962) was used to analyze the data. Table 1 shows the results. Neither the conditions nor rewards are statistically significant for acquisition, indicating that performance was not affected significantly by either magnitude or type of reward.

For extinction, the conditions are not significantly different. The rewards are significant at the .01 level. The order of rewards from most RTE to least RTE is: paper-clip; candy; plastic-token; balloon. (Separate analyses comparing the male and female Ss showed no significant sex differences.) A $t$ test for correlated means was performed comparing the number of correct responses on acquisition during the first 20 trials to the last 20 trials. The resultant t value of 8.72 is significant at the .01 level $(p<.01 ; t=2.32)$. The mean for the last 20 trials was the larger of the two. This appears to eliminate from consideration the interpretation that the seemingly simple task led to the Ss' performing minimally regardles of reward or condition.

The mean difference between the last 20 and first 20 acquisition trials was calculated for both the Token and Direct conditions and a $t$ test comparing the two means was performed. The $t$ of .38 is not significant $(p<.05$; $t=1.64$ ), indicating that the groups were performing in a similar negatively accelerated manner.

\section{Diseussion}

The results appear to partially confirm our hypothesis concerning the differential effects of reward on acquisition and extinction performance. No significant acquisition differences were observed for magnitude or type of reward. It seems reasonable then, to assume that magnitude and type of reward are not significant variables in the acquisition performance of a simple discrimination response by normal first-graders.

The Token-Direct difference on extinction was not statistically significant (though it was in the predicted direction-the Token group showed greater RTE). This
Table 1. Analyses of Variance for Acquisition and Extinction

\begin{tabular}{lrrrccc} 
& \multicolumn{3}{c}{ Acquisition } & \multicolumn{2}{c}{ Extinction } \\
Source & df & \multicolumn{1}{c}{ MS } & F & MS & F \\
Conditions(C) & 1 & 101 & N.S. & 10 & 3.53 \\
Rewards(R) & 3 & 197.6 & N.S. & 13.9 & 4.91 ** \\
CxR & 3 & 85.3 & N.S. & 4 & 1.14 \\
Within Cell & 72 & 214.8 & --- & 2.83 & --- \\
Total & 79 & & & &
\end{tabular}

$* * \mathrm{p}<.01$

result would seem to challenge the view that low level of reward produces greater RTE. It is possible, though, that the Ss did not consider the amount of reward insufficient.

Type of reward, as predicted, did significantly affect extinction performance. It may be that any differential hierarchy of reward preference during acquisition is masked by the major source of variance, KOR (in terms of designation of a specific "correct" choice response). On extinction, however, there is no "correct" response, so the differential effect associated with the previously appropriate response now becomes the major source of variance. Why the particular differential hierarchy is produced is another matter, not essential to this explanation.

\section{Referenees}

HEBB, D. O. Drive and the C.N.S. (conceptual nervous system). Psychol. Rev., 1955, 62, 243-254.

LAWRENCE, D. H., \& FESTINGER, L. Deterrents and reinforcement: The psychology of insufficient reward. Stanford, California: Stanford University Press, 1962.

STEVENSON, H. W., \& WEIR, M. Variables affecting children's performance in a probability learning task. J. exp Psychol. 1959, $57,403-412$.

WINER, B. J. Statistical principles in experimental design. New York: McGraw-Hill, 1962.

\section{Notes}

1. The authors wish to thank Sarah Haycraft for assisting in the running of subjects.

2. Grateful acknowledgement is made to the Board of Education of the City of Louisville for making available the subjects. 